\title{
沸石与酸性水溶液反应的动力学机制 *
}

\author{
张雪䑣 1,2 张荣华 1 \\ ( 1 中国地质科学院矿产资源研究所, 地球化学动力学开放研究实验室, 北京 100037 ; \\ 2 中国地质大学材料科学与工程学院, 北京 100083)
}

\begin{abstract}
摘要 利用连续摚拌筒反应器(CSTR)对天然沸石与酸性水溶液的反应动力学进行研究, 通过改变流速、 $\mathrm{pH}$ 值 等参数, 对反应速率进行计算和比较. 同时利用二次离子质谱(SIMS)、扫描电镜(SEM)对反应后的沸石表面进行 分析研究. 实验结果表明, 沸石中的 $\mathrm{Si} 、 \mathrm{Al} 、 \mathrm{Na}$ 的释放速率在多数情况下不相同, 沸石的溶解为不一致溶解作用. $25{ }^{\circ} \mathrm{C} 、 1.01 \times 10^{5} \mathrm{~Pa}$ 条件下, 硅的释放速率为: 在 $\mathrm{pH}=2.45$ 溶液中反应时, $-r_{\mathrm{S}}=k S\left(a_{\mathrm{H}^{+}}\right)^{125} /\left(a_{\mathrm{S}}\right)^{0.060}$; 在 $\mathrm{pH}=3.26$ 溶液中 反应时, $-r_{\mathrm{Si}}=k S\left(a_{\mathrm{H}^{+}}\right)^{1.50} /\left(a_{\mathrm{Si}}\right)^{0.25}$ ( $S$ 为矿物材料的表面积). SIMS 研究显示, 天然沸石与酸性水溶液的反应中, 在沸石 表面 $\mathrm{Si} 、 \mathrm{Al} 、 \mathrm{Na}$ 在 $100 \mathrm{~nm}$ 的厚度范围内, 随着离表面距离的改变, 在近表面范围内 $\mathrm{Na} 、 \mathrm{Al}$ 大量淋失, 有 $\mathrm{H}^{+}$浸人. 此外, SEM 分析结果显示, 天然沸石与纯水及酸性水溶液反应后, 表面形貌显著不同.
\end{abstract}

关键词：矿物表面, 反应动力学, 不一致溶解, 液/固界面

中图分类号: $\mathrm{O} 643$

沸石族是一类重要的骨架硅酸盐矿物, 近年来 对于它的选择性吸附、离子篮性质和催化性能的研 究较为广泛 ${ }^{[1-4]}$. 同时, 沸石是沉积岩中含量丰富和分 布范围广的矿物之一. 因此, 研究沸石与溶液反应的 液/固界面以及矿物表面, 对于了解溶液中的金属离 子迁移和沉淀有很重要的作用. 研究沸石与水溶液 作用后的固体表面性质变化是表面化学研究中一个 重要的研究方向.

与水溶液反应后的矿物材料表面的性质会发生 变化. 通常出现表面侵蚀、表面吸附、表面离子交换、 新的表面化学物种的形成等. 这些表面变化与水溶 液性质有关, 与水溶液-矿物相间反应动力学过程有 关. 我们 ${ }^{[5-7]}$ 曾对矿物-水溶液非均相反应动力学和矿 物表面变化的研究作过报道. 本文研究了 $25{ }^{\circ} \mathrm{C}$ 、 $1.01 \times 10^{5} \mathrm{~Pa}$ 条件下, 在全混流开放体系内沸石与水 溶液和 $\mathrm{HCl}-\mathrm{H}_{2} \mathrm{O}$ 溶液的反应速率, 以及反应后矿物 表面的变化.

\section{1 实验部分}

\section{1 实验装置}

连续摚拌筒反应器 (continuous stirred tank reactor, CSTR)亦称全混流反应器, 高温、高压 CSTR
是连续搅拌, 连续输人, 连续输出水溶液的反应器. 全混流 CSTR 结构见图 $1^{[5-7]}$. CSTR 常被用于研究固

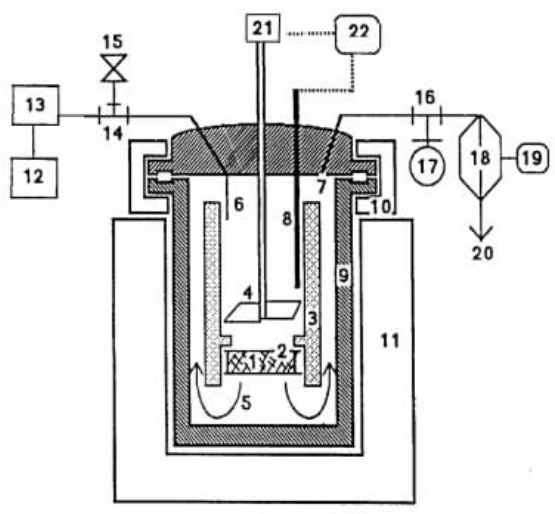

图 1 全混流 CSTR 反应器结构示意图

Fig.1 The schematic illustration of the hydrothermal mixed flow reactor system and the across section of the reactor vessel

1) sample position; 2) nylon net; 3) sample shelf; 4) lamina; 5) cycle of water; 6) inlet; 7) outlet; 8) thermalcouple; 9) high pressure vessel; 10) half ring; 11) furnace; 12) reservoir; 13) high pressure pump; 14, 16) connection; 15) safe disk; 17) pressure gauge; 18) back pressure regulator; 19) gas cylinder; 20) sample outlet; 21)magnetic stirrer housing; 22) temperature and stirring controller

2004-10-14 收到初稿, 2004-12-20 收到修改稿. 联系人:张雪肜(E-mail:zrhhsm@pku.edu.cn; Tel:010-68329535). *国家科技部基础性 工作专项(2001DEA30041, 2002DEA30084, 2003DEA2C021)和国家自然科学基金(20373064)资助项目 
体与溶液的反应 ${ }^{[8-9]}$. 还有人 ${ }^{[10]}$ 用 CSTR 研究地热区 的深部热水和表层水混合的化学反应和地热利用.

\section{2 样品准备}

样品选择天然沸石, 产于新疆伟晶岩脉内. 将样 品粉碎, 过篮, 超声波除去表面粉尘, 然后在尼龙样 品袋内装好, 放人反应器内, 尼龙布的孔隙为 300 目. 矿物粒径分为 $20 \sim 40 、 40 \sim 60 、 60 \sim 80$ 目三种. 天 然沸石的化学分析结果见表 1 . 由表 1 可知, 该天然 沸石为标准的钠沸石.

\section{3 实验方法}

使用 CSTR 时, 可以改变和调控温度、压力、流 速、搅拌速率、溶液性质、矿物表面积、反应筒里固体 样品和液体的体积比等各种变量. 进行稳态操作时, 使反应器恒温, 用背压控制器(图 1-18)固定压力, 使 用压力液泵(图 1-13)保持反应体系在恒温下固定流 速. 实验温度可以精确控制 $\left( \pm 1{ }^{\circ} \mathrm{C}\right)$. 可以在反应过程 中不断地变化输人溶液. 实验中可变换输人不同 $\mathrm{pH}$ 值的溶液, 但是在不停顿的流动体系内, 改换一种新 输人溶液一般要等待 $1 \mathrm{~h}$ 之后(与流速大小有关), 使 反应体系趋于稳态后再取样.

当反应装置安装好, 装人沸石样品(1.0 1.3 g), 启 动洜进行实验之后, 一方面输人未反应的溶液; 同 时, 反应后的溶液立即输出. 一般在 $25{ }^{\circ} \mathrm{C}$ 时等待 24 $\mathrm{h}$ 才开始取样. 通常认为晶体表面有极细小粉尘, 初 始的反应速率会很高. 并且, 反应初始阶段, 晶体表 面能高, 也是反应速率很高的原因之一. 我们在实际 收集各阶段实验样品时也发现, 最初阶段反应速率 较大的现象. 液体样品不间断地由反应器流出来, 当 确认反应速率稳定之后, 在恒温、恒压下的每个流速 点上一般取三个样品. 每次取样约 $10 \mathrm{~mL}$, 进行样品 分析(分析仪器 ICPMS, 精度到 $10^{-9}$ ). 实验在 $25{ }^{\circ} \mathrm{C}$ 、 $1.01 \times 10^{5} \mathrm{~Pa}$ 条件下, 通常, 一次反应动力学实验需 连续进行 500 600 h. 搅拌速率为 $100 \sim 105 \mathrm{r} \cdot \mathrm{min}^{-1}$. 搅拌速率影响溶解反应速率, 本文选择的搅拌速率 是为了研究远平衡条件下的溶解实验 ${ }^{[5,11}$.

\section{2 结果与讨论}

\section{1 沸石与水溶液及酸性溶液反应的动力学}

在 CSTR 中输运过程的物质平衡可用式(1)表 示 ${ }^{[5-7}:$

$$
\mathrm{d} U_{i} / \mathrm{d} t=r v_{i} S / V-F / V\left(U_{i}-U_{i}^{0}\right)
$$

式中 $U_{i}^{0}$ 为溶液中物种 $i$ 的初始浓度, $U_{i}$ 为物种 $i$ 的 输出浓度, $S$ 为沸石表面积, $V$ 为压力容器的体积, $F$ 为流速, $r$ 为反应速率, $v_{i}$ 为化学计量系数. 输出溶液 通过一个电导检测器, 在反应系统中, 流体电导的变 化可显示出系统是否处于一个稳态或非稳态. 当 $\mathrm{d} U_{i} / \mathrm{d} t=0$, 表示系统处于稳态, 可以取输出溶液样品.

在流动体系中使用低 $\mathrm{pH}$ 值酸性溶液和中性去 离子纯水, 在不同流速下, 反复测量沸石的溶解速 率. 结果显示, 沸石的溶解速率随着输人溶液的 $\mathrm{pH}$ 值、输出溶液中 $\mathrm{Si} 、 \mathrm{Al} 、 \mathrm{Na}$ 的可溶性物种浓度和流速 的变化而改变.

根据测量的反应体系中输出浓度与流速的关系 (式 1)可以计算 $\mathrm{Na} 、 \mathrm{Al} 、 \mathrm{Si}$ 在水溶液中的溶解反应速 率. 沸石的溶解过程可以用各种流速下输出溶液浓 度与速率的相互关系表述, 也可用各元素释放速率 比加以描述. 一般来说, 溶解速率随着流速的增加而 增大. 为了节省篇幅, 我们未列出反应速率与流速的 关系图. 然而, 在一个恒定流体动力学状态下 (流速不 变), 溶解速率随着 $\mathrm{pH}$ 值降低而增大, 见图 2. 当输 人溶液 $\mathrm{pH}$ 值降低时, 溶解速率增大 ; 当流速增大 时, 溶解速率也增大. 同时, $\mathrm{Si} 、 \mathrm{Al} 、 \mathrm{Na}$ 的可溶性物种 浓度的增大, 经常导致溶解速率下降, 或者说, 阻止 反应速率的进一步增加. 许多硅酸盐溶解时都有这 种现象 ${ }^{[12]}$.

沸石溶解过程中对 $\mathrm{pH}$ 值很强的依赖性及受可 溶性物种浓度的影响可用式(2)表示 ${ }^{[6]}$ :

$$
-r=k S\left(a_{\mathrm{H}^{+}}\right)^{m} /\left(a_{i}\right)^{n}
$$

式中 $-r$ 为溶解速率, $k$ 为速率常数, $S$ 为矿物材料的 表面积, $m$ 和 $n$ 是速率指数, $a_{\mathrm{H}^{+}}$表示溶液中 $\mathrm{H}^{+}$活度. 对 $\mathrm{Si} 、 \mathrm{Al} 、 \mathrm{Na}$ 的释放速率来说, 在 $\mathrm{pH}=2.45$ 的溶液 中, $m_{\mathrm{Si}} 、 m_{\mathrm{Al}} 、 m_{\mathrm{Na}}$ 分别为 $1.25 、 0.78 、 1.00$; 在 $\mathrm{pH}=3.26$ 的溶液中, $m_{\mathrm{Si}} 、 m_{\mathrm{Al}} 、 m_{\mathrm{Na}}$ 分别为 $1.50 、 1.88 、 1.69 . a_{i}$ 为 水化物种 $i$ 的活度, 例如 $i$ 可以是溶液中的 $\mathrm{Si} 、 \mathrm{Al}$ 、 Na.

$\lg r-\lg c$ 图(图 3)表示 $\mathrm{Si} 、 \mathrm{Al}$ 和 $\mathrm{Na}$ 的反应速率

表 1 天然沸石化学分析结果 $(w(\%))$

Table 1 The chemical compositions of the natural zeolite

\begin{tabular}{cccccccccccc}
\hline $\mathrm{SiO}_{2}$ & $\mathrm{Al}_{2} \mathrm{O}_{3}$ & $\mathrm{Fe}_{2} \mathrm{O}_{3}$ & $\mathrm{FeO}$ & $\mathrm{MgO}$ & $\mathrm{K}_{2} \mathrm{O}$ & $\mathrm{Na}_{2} \mathrm{O}$ & $\mathrm{TiO}_{2}$ & $\mathrm{MnO}$ & $\mathrm{P}_{2} \mathrm{O}_{5}$ & $\mathrm{H}_{2} \mathrm{O}$ & $\mathrm{CO}_{2}$ \\
\hline 46.75 & 26.82 & 0.04 & 0.07 & 0.19 & 0.20 & 15.88 & 0.01 & 0.09 & 0.10 & 9.27 & 0.24 \\
\hline
\end{tabular}



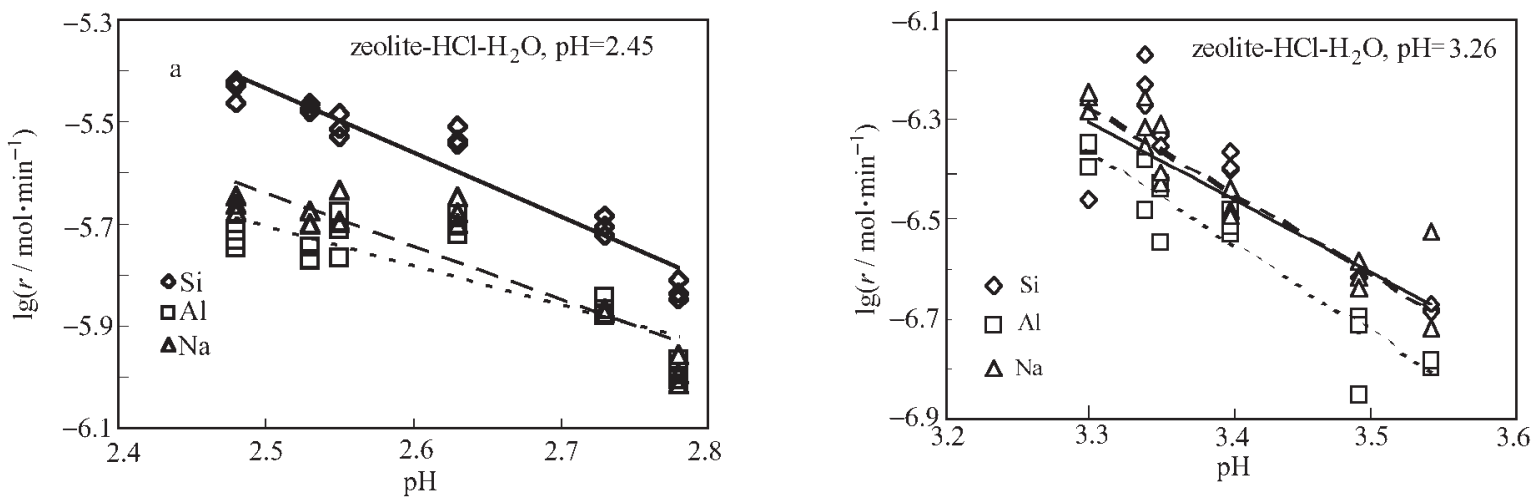

图 2 沸石的溶解速率对数值与输出溶液 $\mathbf{p H}$ 关系图

Fig.2 Logarithm of the dissolution rates of zeolite $v s$ the $\mathrm{pH}$ of the output solutions during zeolite reacted with low $\mathrm{pH}$ solutions

Solid lines represent logarithm of the dissolution rates of Si vs the $\mathrm{pH}$ of the output solutions; dashed lines represent logarithm of the dissolution rates of $\mathrm{Na} v s$ the $\mathrm{pH}$ of the output solutions; dotted lines represent logarithm of the dissolution rates of $\mathrm{Al} v s$ the $\mathrm{pH}$ of the output solutions.

(释放速率) 及与反应产物输出浓度的关系. 图 $3 \mathrm{a} 、 3 \mathrm{~b}$ 和 $3 \mathrm{c}$ 的共同特征为总反应速率受反应产物输出浓 度的影响. 当输出浓度升到足够高时, 反应速率呈现 下降趋势. 如 $\mathrm{Al}$ 和 $\mathrm{Na}$ 浓度不高时, 对反应速率的 影响较小.

远平衡态状态下, 溶解速率是通过它与 $a_{i}$ 的线 性关系获得的. 因为溶解的不一致性, 在多数情况 下, $\mathrm{Si}$ 的反应速率不同于 $\mathrm{Al}$ 和 $\mathrm{Na}$ 的反应速率. 速 率公式(2)中 $\mathrm{Si}$ 的反应速率指数 $(n)$ 也与 $\mathrm{Al}$ 或 $\mathrm{Na}$ 的不同. 沸石在 $\mathrm{pH}=2.45$ 溶液里的溶解过程可用图 3 表示. 沸石在 $\mathrm{pH}=2.45$ 和 3.26 溶液里的溶解过程 中 $n_{\mathrm{Si}} 、 n_{\mathrm{A} 、} 、 n_{\mathrm{Na}}$ 分别为 $0.60 、 0.25 、 0.40$ 和 $0.25 、 0.68$ 、 0.62 .

根据溶解速率 $(\mathrm{Na} 、 \mathrm{Al} 、 \mathrm{Si}$ 的反应速率) 与输出 溶液浓度之间的关系(见图 3)及溶解速率对数值与 $\mathrm{pH}$ 值之间的关系(图 2)可以获得沸石的溶解速率方 程.

一般,简化的速率方程可以表示为 ${ }^{[13]}$ :

$$
\begin{aligned}
& -r=k \prod a_{i}^{n_{i j}}[1-\exp (-A / \sigma R T)] \quad \text { (近平衡) } \\
& -r=k \prod a_{i}^{n_{i j}} \quad \text { (远平衡) }
\end{aligned}
$$

式中 $-r$ 是反应速率, $a_{i}$ 是水化物种 $i$ 的活度, $n_{i j}$ 代表 第 $j$ 次反应的化学计量系数, $A$ 为化学亲合势, $\sigma$ 是 化学计量数. 在实验过程中保持高流速, 开放体系处 于远平衡状态. 事实上, $-\exp (-A / \sigma R T)$ 的数值在远 平衡状态时可忽略不计.

实际获得的溶解速率, 如硅的释放速率为

在 $\mathrm{pH}=2.45$ 的溶液中反应时

$$
\begin{aligned}
& -r_{\mathrm{Si}}=k S\left(a_{\mathrm{H}^{+}}\right)^{1.25} /\left(a_{\mathrm{Si}}\right)^{0.60} \\
& -r_{\mathrm{Si}}=k S\left(a_{\mathrm{H}^{+}}\right)^{0.78} /\left(a_{\mathrm{Si}}\right)^{0.25} \\
& -r_{\mathrm{Si}}=k S\left(a_{\mathrm{H}^{+}}\right)^{1.00} /\left(a_{\mathrm{Si}}\right)^{0.40}
\end{aligned}
$$

在 $\mathrm{pH}=3.26$ 的溶液中反应时

$$
\begin{aligned}
& -r_{\mathrm{Si}}=k S\left(a_{\mathrm{H}^{+}}\right)^{1.50} /\left(a_{\mathrm{Si}}\right)^{0.25} \\
& -r_{\mathrm{Si}}=k S\left(a_{\mathrm{H}^{+}}\right)^{1.58} /\left(a_{\mathrm{Si}}\right)^{0.68} \\
& -r_{\mathrm{Si}}=k S\left(a_{\mathrm{H}^{+}}\right)^{1.69}\left(a_{\mathrm{Si}}\right)^{0.62}
\end{aligned}
$$
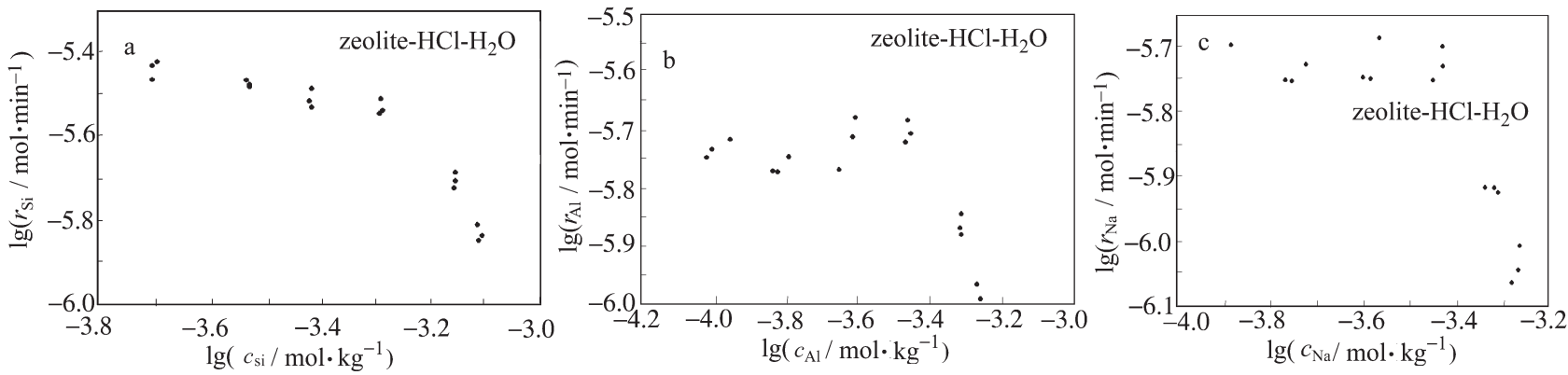

图 3 沸石与 $\mathrm{pH}=2.45$ 的 $\mathrm{HCl}-\mathrm{H}_{2} \mathrm{O}$ 溶液反应时, 反应速率与浓度的关系

Fig.3 Variation of release rate with concentration when zeolite reacted with $\mathrm{HCl}-\mathrm{H}_{2} \mathrm{O}$ solution of $\mathrm{pH}=2.45$ 

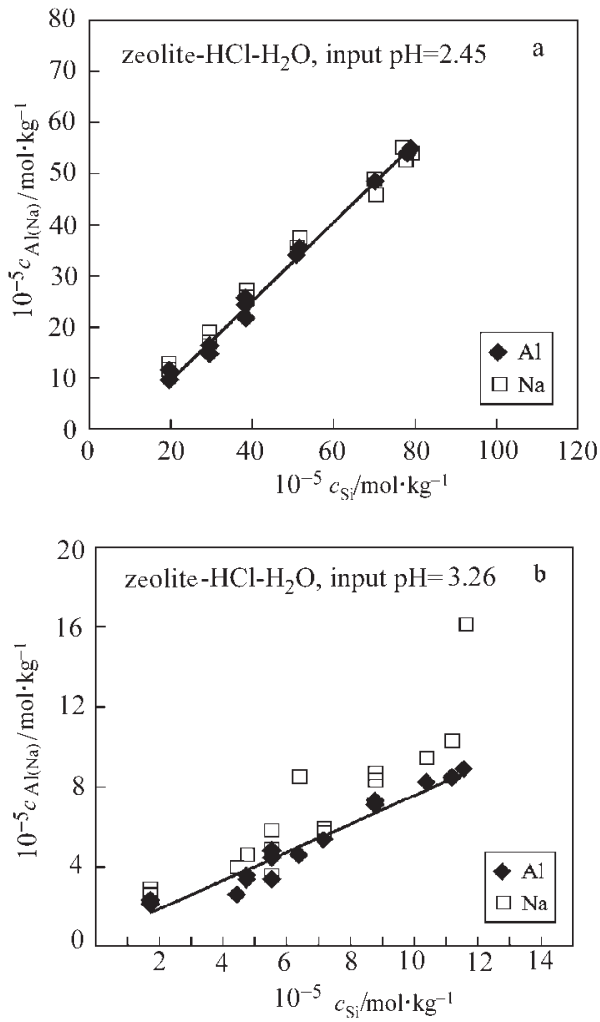

图 4 沸石与 $\mathrm{HCl}-\mathrm{H}_{2} \mathrm{O}$ 溶液反应时 $\mathrm{Al} 、 \mathrm{Na}$ 浓度与 $\mathrm{Si}$ 浓度 的关系

Fig.4 Molar concentration of $\mathrm{Al}$ or $\mathrm{Na}$ as function of molar concentration of $\mathrm{Si}$ when zeolite reacted with $\mathrm{HCl}-\mathrm{H}_{2} \mathrm{O}$

(5-1)和(5-2)两组方程形式相同, 适用于不同 $\mathrm{pH}$ 溶 液里的溶解反应. 输人溶液的 $\mathrm{H}^{+}$活度影响速率的 $m$ 与 $n$ 数值, 所以只能在有限的 $\mathrm{pH}$ 值范围内获得速 率公式.

\section{2 沸石的不一致溶解过程与 SIMS 分析}

根据溶解后输出溶液中的 $\mathrm{Al} / \mathrm{Si}$ 和 $\mathrm{Na} / \mathrm{Si}$ 摩尔 比与固体中 $\mathrm{Al} / \mathrm{Si}$ 和 $\mathrm{Na} / \mathrm{Si}$ 摩尔比的比较, 证实沸石 的溶解为不一致溶解作用 ${ }^{[14]}$. 实验用的未反应的沸 石 $\mathrm{Al} / \mathrm{Si}$ 和 $\mathrm{Na} / \mathrm{Si}$ 摩尔比分别为 0.67 和 0.66 , 固体 内 $\mathrm{Al} / \mathrm{Na}$ 摩尔比是 $1: 1$. 但是, 实验结果表明, 反应后 的溶解产物中 $\mathrm{Al} / \mathrm{Si}$ 和 $\mathrm{Na} / \mathrm{Si}$ 摩尔比均比固体中的 高, 说明 $\mathrm{Na} 、 \mathrm{Al}$ 的释放速率要大于 $\mathrm{Si}$ 的释放速率, $\mathrm{Na} 、 \mathrm{Al} 、 \mathrm{Si}$ 的释放速率不同.

图 3 显示在 $\mathrm{Al}$ 和 $\mathrm{Na}$ 的低浓度时, $\mathrm{Si} 、 \mathrm{Al}$ 和 $\mathrm{Na}$ 的释放速率不同, $\mathrm{Si}$ 和 $\mathrm{Al} 、 \mathrm{Na}$ 不是以同一反应速率 进人溶液. 实验研究证实了沸石在水溶液中的溶解 通常为不一致溶解. 溶液的 $\mathrm{pH}$ 值变化会强烈影响 溶解不一致过程, 在 $\mathrm{pH}=2.45$ 溶液里 $\mathrm{Na} / \mathrm{Si}$ 和 $\mathrm{Al} / \mathrm{Si}$ 的摩尔比相同, 但在 $\mathrm{pH}=3.26$ 溶液里, $\mathrm{Na} / \mathrm{Si}$ 和 $\mathrm{Al} / \mathrm{Si}$
的摩尔比是不同的(见图 4).

不一致溶解通常是由于在矿物-水反应中矿物 表面淋失层和表面吸附物或次生矿物的变化引起 的 ${ }^{[15]}$. 图 5 为沸石表面 SIMS 研究结果, 横坐标是溅 射的累积时间, 表示的是距离表面的深度. 可以看 出, 反应后的表面层内物质成分是不均匀的. 新鲜沸 石(图 5a)和与水反应后的沸石(图 5b)比较, 发现两 者的 $\mathrm{Al} 、 \mathrm{Na}$ 含量明显不同. 反应后沸石表层的 $\mathrm{Al}$ 、 $\mathrm{Na}$ 的计量数波动变化, 其中近表层 $\mathrm{Na}$ 的计量数明 显降低.

沸石与 $\mathrm{HCl}-\mathrm{H}_{2} \mathrm{O}$ 溶液反应后, 近表面层内有 $\mathrm{H}^{+} 、 \mathrm{Cl}^{-}$渗人. $\mathrm{Al} 、 \mathrm{Na}$ 的含量比新鲜的沸石含量更低. 并且, 按由表及里顺序 $\mathrm{Si} 、 \mathrm{Al} 、 \mathrm{Na} 、 \mathrm{H}^{+} 、 \mathrm{Cl}^{-}$的含量波 动更大. 此现象表明, 溶解过程中固体表层里物质重 新分配. 图 $5 \mathrm{c}$ 和图 $5 \mathrm{~b}$ 相比, $\mathrm{H}^{+} 、 \mathrm{Cl}^{-}$波动更大, $\mathrm{H}^{+} 、$ $\mathrm{Cl}^{-}$渗人是明显增加的.

这种反应后的表层内各个离子的含量变化是造 成反应体系的宏观的化学动力学过程的根源, 过去 作者有过研究 ${ }^{[16]}$.

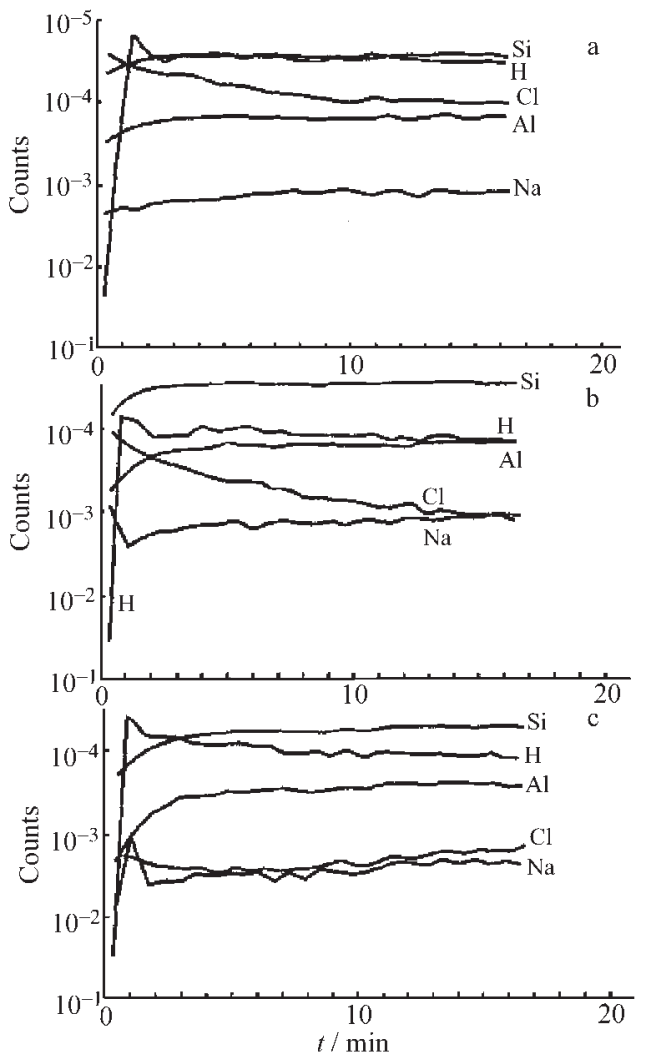

图 5 沸石表面 SIMS 研究

Fig.5 SIMS study of zeolite surface

(a) unreacted; (b) after reacted with water;

(c) after reacted with acidic solutions 

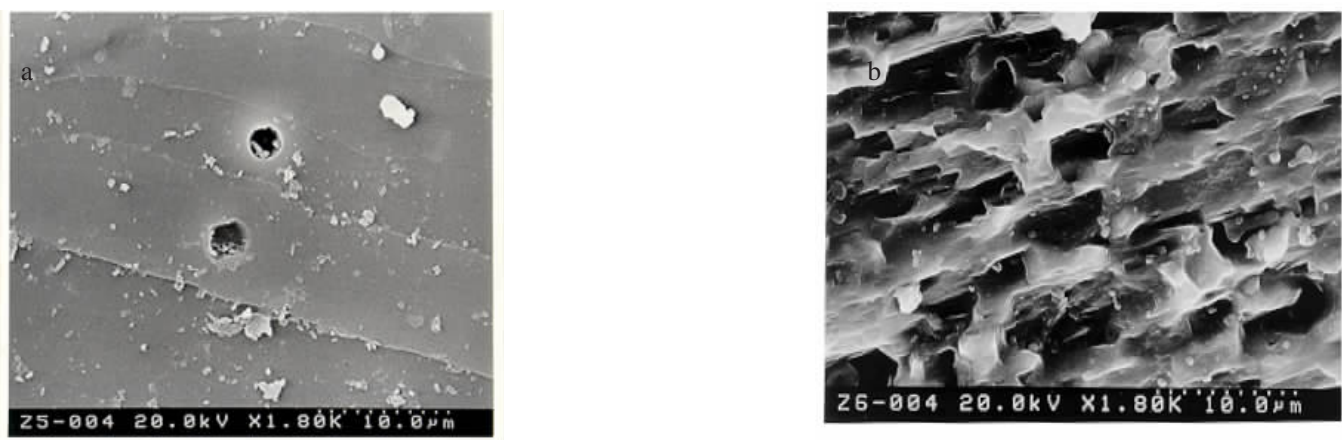

图 6 沸石与水溶液 (a)及 $\mathrm{pH}=\mathbf{2 . 4 5}$ 的 $\mathrm{HCl}-\mathrm{H}_{2} \mathrm{O}$ 溶液 (b) 反应后的 SEM 照片

Fig.6 The SEM images of zeolite surface after reacted with water (a), and $\mathrm{HCl}-\mathrm{H}_{2} \mathrm{O}$ solution of $\mathrm{pH}=2.45(\mathrm{~b})$

\subsection{SEM 分析}

图 6 为 $25^{\circ} \mathrm{C}$ 下沸石与水溶液及 $\mathrm{pH}=2.45$ 的酸 性溶液反应后的 SEM 照片. 比较图 $6 \mathrm{a}$ 与图 $6 \mathrm{~b}$ 发 现, $25{ }^{\circ} \mathrm{C}$ 下与水反应的流动体系, 尤其在快速流动 条件下, 反应体系处于远平衡态下, 这时表面的侵蚀 坑稀少, 随机发生, 形成不规则形态, 孔隙较大, 而且 稀疏(见图 6a).

当沸石与电解质溶液反应时, 如与低 $\mathrm{pH}$ 值的 酸性水溶液的反应情形下, 反应是通过 $\mathrm{H}^{+}$渗人表面, 在这一过程发生离子交换. 这时反应速率往往与反 应产物浓度和 $\mathrm{H}^{+}$活度有关. 这时表面侵蚀坑个体的 面积小, 但广泛均匀地出现. 并且表面侵蚀具有晶体 的结构形态(见图 6b). 显然, 水分子对表面的作用与 $\mathrm{H}^{+}$对表面的作用机制是不同的 ${ }^{[2]}$.

研究沸石与不同酸性溶液的溶解反应及其表面 变化, 有助于从理论上认识铝硅酸盐在溶液里溶解 反应的影响因素. 研究结果显示出一个普遍规律, 即 溶液的 $\mathrm{H}^{+}$活度增加导致溶解速率增加, 并通常表现 为速率与 $\mathrm{H}^{+}$活度的分数数值有密切关系 ${ }^{[12,17-18]}$. 同 时, 溶液里的 $\mathrm{Si} 、 \mathrm{Al}$ 和 $\mathrm{Na}$ 的浓度增加阻止溶解反 应. 从应用方面看, 沸石在化工、环境工程中用途很 大, 但是, 使用者对于使用时沸石自身的溶解和表面 变化注意不多. 为此, 本文的实验结果会给出使用时 要注意的问题.

\section{3 结 论}

沸石在多数情况下是不一致溶解过程. 溶解速 率随着 $\mathrm{pH}$ 值降低而增大. 当输入溶液 $\mathrm{pH}$ 值降低 时, 溶解速率增大 ; 当流速增大时, 溶解速率也增大. 同时, $\mathrm{Si} 、 \mathrm{Al} 、 \mathrm{Na}$ 的可溶性物种浓度的增大, 经常导致 溶解速率下降, 或者说, 阻止反应速率的进一步增加.
$25{ }^{\circ} \mathrm{C} 、 1.01 \times 10^{5} \mathrm{~Pa}$ 条件下, 硅的释放速率为: 在 $\mathrm{pH}=2.45$ 的溶液中反应时: $-r_{\mathrm{Si}}=k S\left(a_{\mathrm{H}^{+}}\right)^{1.25} /\left(a_{\mathrm{Si}}\right)^{0.60}$ 在 $\mathrm{pH}=3.26$ 溶液中反应时: $-r_{\mathrm{Si}}=k S\left(a_{\mathrm{H}^{+}}\right)^{1.50} /\left(a_{\mathrm{Si}}\right)^{0.25}$

铝和钠的释放速率与硅的释放速率形式相似.

使用 CSTR 开放-流动体系研究矿物与水溶液 反应, 以沸石为例, 对同一样品一方面研究其反应动 力学过程, 另一方面对应 SEM 研究表面形态, 用 SIMS 方法研究反应前后表层成分变化. 沸石与 $\mathrm{HCl}-\mathrm{H}_{2} \mathrm{O}$ 溶液反应后, 近表面层内有 $\mathrm{H}^{+} 、 \mathrm{Cl}^{-}$渗人. $\mathrm{Al} 、 \mathrm{Na}$ 的含量比新鲜的沸石含量更低.

研究发现矿物材料的表面化学是影响矿物/水 溶液相间化学动力学过程的关键因素. 矿物表面在 矿物水溶液相互作用中不断发生变化. 这种表面变 化与矿物在水溶液内反应速率和反应控制等因素有 关, 与 $\mathrm{H}^{+}$浓度大小有关.

\section{References}

1 Zhang, M.J.;Zheng, A.M.; Deng, F.; Yue, Y.; Ye, Z.H. Science in China B, 2002, 32(6):509 [张铭金, 郑安民, 邓 风, 岳 勇, 叶朝辉.中国科学 B(Zhongguo Kexue B), 2002, 32(6): 509]

2 Justel, T.; Wiechert, D.U.; Lau, C. Advanced Functional Materials, 2001, 11(2): 105

3 Jolly, S.; Saussey, J.; Bettahar, M.M.; Lavalley, J.C.; Benazzi, E. Applied Catalysis A: General, 1997, 156(1): 71

4 Wang, X.H.;Liu, Y.L.; Song, C.Y.; Huang, L.H;Zhang, J.X. Chinese Journal of Inorganic Chemistry, 2003, 19(8) : 819 [王晓华, 刘应亮, 宋春燕, 黄浪欢, 张静㛠. 无机化学学报 (Wuji Ниахие Хиеbаo), 2003, 19(8):819]

5 Zhang, R.H.; Hu, S.M.; Crerar, D. Chemical kinetics studies of 
minerals in hydrothermal systems and mass transfer. Beijing: Science Press, 1992:15 [张荣华, 胡书敏, D. Crerar. 矿物在热 液内的化学动力学和物质迁移. 北京:科学出版社, 1992:15]

6 Zhang, R.H.; Hu, S.M. Science in China D, 1996, 39(6):561 [张荣华, 胡书敏. 中国科学 D (Zhongguo Kexue D), 1996, 39(6): $561]$

7 Zhang, X.T.; Zhang, R.H.; Hu, S.M.; Yu, W.B. Acta Geologica Sinica, 2000, 74(2): 406

8 Dove, P.M; Crerar, D. Geochimica et Cosmochimica Acta, 1990, 54(4): 955

9 Rimstidt, J.D.; Dove, P.M. Geochimica et Cosmochimica Acta, 1986, $50: 2509$

10 Henley, R.W. Review of Economic Geology, 1985, 1:177
11 Metz, V.; Ganor, J. Geochimica et Cosmochimica Acta, 2001, 65 (20): 3475

12 Oelker, E.H.; Schott, J.; Davidal, J. L. Geochimica et Cosmochimica Acta, 1994, 58: 2011

13 Aagaard, P.; Helgeson, H.C. American Journal of Science, 1982, $282: 237$

14 Hellmann, R.; Crerar, D.; Zhang, R.H. Solid State Ionics, 1989, $32 / 33: 314$

15 Hochella, Jr. M. F.; White, A. F. Mineralogy review.Washingto, D.C.: Mineralogical Society of America, 1989: 23

16 Zhang, R.H.; Hu, S.M., Proceeding of 30th IGC. Vol. 19. Beijing: Geological Publishing House, 1996: 29

17 Oelker, E.H. Geochimica et Cosmochimica Acta, 2001, 65:3703

18 Zhang, R.H.; Hu, S.M.; Su, Y.F. Acta Geologica Sinica, 2002, 76(3): 351

\title{
Kinetics Study of Zeolite in Hydrothermal Solutions*
}

\author{
ZHANG, Xue-Tong ${ }^{1,2}$ ZHANG, Rong-Hua ${ }^{1}$ \\ ( ${ }^{1}$ Open Research Laboratory of Geochemical Kinetics, Chinese Academy of Geological Sciences, Beijing 100037 ; \\ ${ }^{2}$ School of Material Science and Technology, China University of Geosciences, Beijing 100083)
}

\begin{abstract}
In this paper, we conducted a series of experiments on zeolite in water and in acidic solutions using a continuous stirred tank reactor (CSTR) of an open system. We measured the reaction rates for zeolite with water and with low $\mathrm{pH}$ solutions at room temperature, and conducted associated studies of mineral surfaces by using SEM and SIMS. The results showed that incongruent dissolution of zeolite was related to the surface chemical modifications.
\end{abstract}

Keywords: Mineral surface, Reaction kinetics, Incongruent dissolution, Liquid /solid interface 